\title{
Tips for Office-Based Transurethral Biopsy and Fulguration as a Treatment of Tiny Bladder Tumors*
}

\author{
Teiichiro Aoyagi $^{1 \#}$, Isao Kuroda ${ }^{1}$, Masaaki Tachibana ${ }^{2}$ \\ ${ }^{1}$ Tokyo Medical University, Ibaraki Medical Center, Inashiki, Japan \\ ${ }^{2}$ Tokyo Medical University, Tokyo, Japan \\ Email: "aoyagite@tokyo-med.ac.jp
}

Received August 8, 2013; revised September 6, 2013; accepted September 14, 2013

Copyright (C) 2013 Teiichiro Aoyagi et al. This is an open access article distributed under the Creative Commons Attribution License, which permits unrestricted use, distribution, and reproduction in any medium, provided the original work is properly cited.

\begin{abstract}
Among the patients who underwent outpatient cystoscopy as a follow up of bladder cancer, quite a few patients are observed tiny papillary lesions suspicious for tumor recurrence. Transurethral biopsy and/or resection under spinal or general anesthesia in a hospitalized setting are the usual procedures for this kind of patients, even though these procedures are simple and brief. We tried transurethral biopsy and fulguration as a treatment for very small bladder tumor in an outpatient setting and here describe tips for these procedures. Olympus CYF-VA flexible cystoscope, a 3 Fr. diathermy probe, monopolar electrosurgical unit were used. No additional anesthetics except for $10 \mathrm{ml}$ of $2 \%$ Xylocaine gel applied to (male patient's) urethra as an initial flexible cystoscopic procedure, was required for tumor treatment. Distilled water was used as an irrigation fluid. Experienced tips of the procedures to avoid tumor recurrence are as follows: tumor should be one location, size of the tumor should be less than $5 \mathrm{~mm}$, bladder should be washed several times after the fulguration with hundreds $\mathrm{ml}$ of distilled water. We conclude that outpatient biopsy and fulguration for tiny bladder tumor is effective and less invasive procedure as a treatment of bladder cancer patients.
\end{abstract}

Keywords: Transurethral Surgery; Bladder Cancer; Office Urology; Fulguration; Biopsy

\section{Introduction}

Office-based cystoscopic examination is a usual established method as an initial and postoperative surveillance of bladder tumor [1]. During the follow up period, small, low grade and superficial bladder tumor recurs in quite a few patients [2]. Although experience and efficacy of office-based fulguration for low grade papillary lesions has been reported previously, this procedure does not performed routinely in the clinical practice, presumably because there is no precise explanation on actual methodology and safety [3-5]. However, considering increasing comorbidities, use of anti-coagulation medication, economic restriction and so on, ambulatory treatment for these lesions should be beneficial both for patients and surgeons [6]. Here, we present the tips of ambulatory biopsy and fulguration treatment for tiny bladder papillary lesions with some improvements after experiences of failure cases.

\footnotetext{
*The authors declare that they have no conflict of interests, no funds and no grant on this project.

${ }^{\#}$ Corresponding author.
}

\section{Patients and Methods}

Fourteen patients, 19 procedures were enrolled in this report. The patient profiles are listed in the Table 1. All the patients had the history of transurethral resection (TUR) or nephroureterectomy surgery under spinal or general anesthesia previously, and followed with repeat surveillance flexible cystoscopy in an outpatient setting. Safety to use diathermia for these patients was confirmed by previous surgery, and informed consent on the treatment of ambulatory procedures was made by patient's signature. This report does not violate ethical standards of the Declaration of Helsinki and its revisions. No additional anesthetics except for $10 \mathrm{ml}$ of $2 \%$ Xylocaine gel applied to (male patient's) urethra as an initial flexible cystoscopic procedure, was required for tumor treatment. Olympus (Tokyo, Japan) CYF-VA flexible cystoscope and Versa Pro unit, Takei (Tokyo Japan) monopolar electrosurgical unit B-1, Olympus flexible biopsy forceps and 3 Fr. Takei Bugbee ureteroscope electrode were used. Normal saline was used for observation, and distilled water was used for treatment as irrigation fluid (Figure 1). 
Table 1. Patient profiles and results.

\begin{tabular}{|c|c|c|c|c|c|c|}
\hline $\mathrm{Pt}$ & Age & Previous TUR & Tumor & Pathoogy & Result & Comment \\
\hline \multirow[t]{2}{*}{1} & 71 & $6 \mathrm{~m}$ & Dome 1 trigonum 3 & UC G1 & 3 m relapse & Treated with re-TUR \\
\hline & 72 & $1 \mathrm{y}$ & Dome 1 & UC G1 & $4 \mathrm{y}$ free & \\
\hline \multirow[t]{2}{*}{2} & 82 & Primary & Trigonum 3 & UC G1 & 4 m relapse & Treated with office-based re-TUF \\
\hline & 82 & $4 \mathrm{~m}$ & Near left orifice 1 & UC G1 & $4 \mathrm{y}$ free & \\
\hline \multirow[t]{4}{*}{3} & 77 & $3 \mathrm{~m}$ & Trigonum 1 & UC G2 & $3 \mathrm{~m}$ relapse & Treated with office-based re-TUF \\
\hline & 78 & $3 \mathrm{~m}$ & Bladder neck 1 & & 9 m relapse & Treated with re-TUR \\
\hline & 79 & $7 \mathrm{~m}$ & Trigonum 1 & & 10 m relapse & Treated with office-based re-TUF \\
\hline & 80 & $10 \mathrm{~m}$ & Trigonum 1 & & $6 \mathrm{~m}$ free & Severe Alzheimer disease \\
\hline 4 & 60 & $8 \mathrm{y}$ & Dome $1 \mathrm{~cm} \times 2$ & UC G2 & $5 \mathrm{~m}$ relapse & HD patient, Treated with re-TUR \\
\hline 5 & 83 & $6 \mathrm{~m}$ & Left orifice 1 & UC G2 & $2 \mathrm{~m}$ relapse & Other location treated with re-TUR \\
\hline 6 & 80 & $6 \mathrm{~m}$ & Left wall 1 & UC G1 & 5 m relapse & Other location treated with re-TUR \\
\hline 7 & 62 & $3 \mathrm{~m}$ & Left wall 1 & & $3 \mathrm{~m}$ relapse & Other location treated with re-TUR \\
\hline 8 & 76 & $3 y$ & Right wall 1 & UC G1 & $4 \mathrm{y}$ free & \\
\hline 9 & 71 & $1 \mathrm{y}$ & Bladder neck 1 & UC G1 > G2 & $4 y$ free & \\
\hline 10 & 66 & $2 y$ & Anterior wall 1 & & $2 \mathrm{y}$ free & \\
\hline 11 & 81 & $2 \mathrm{y}$ & Trigonum 1 & & $2 \mathrm{y}$ free & \\
\hline 12 & 68 & $6 \mathrm{~m}$ & Bladder neck 1 & UC G2 & $7 \mathrm{~m}$ free & \\
\hline 13 & 71 & $1 \mathrm{y}$ & Anterior wall 1 & & $6 \mathrm{~m}$ free & \\
\hline 14 & 69 & $16 \mathrm{~m}$ & Bladder neck 1 & UC G1 & 2.5 y free & Post nephroureterectomy \\
\hline
\end{tabular}

m: month, y: year, re-TUR: Transurethral resection of bladder tumor after admission, HD: hemodialysis, UC: urothelial carcinoma.

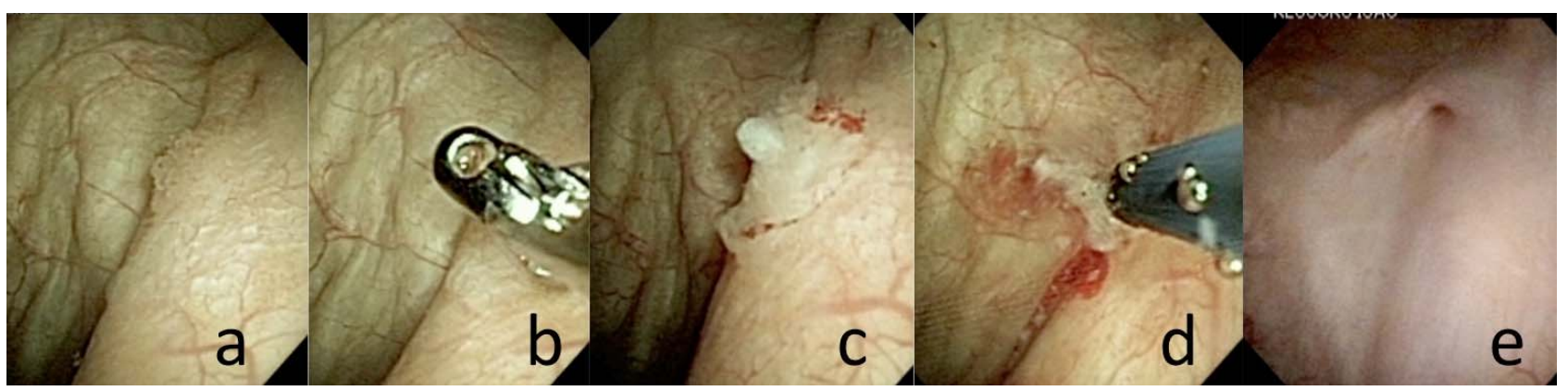

Figure 1. Examples of biopsy and TUF (\#2 patient, second procedure of biopsy and TUF, (a), (b), (c), (d). Cystoscopy after 12 months showed no recurrence, $(e))$.

\section{Experiences}

The first patient ( $\mathrm{Pt} \# 1)$ had recurrent small tumor around the fulgurated tiny tumors after 3 months, presumably due to multiplicity of tumors and insufficient irrigation after treatment. Second patient, had maintenance hemo-

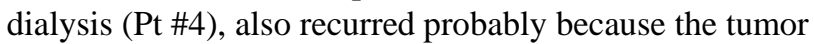
was too big (about $1 \mathrm{~cm} \times 2$ ) to treat in this setting. So in treating the following case, we indicated this procedure for a sole papillary lesion. In addition, after the fulguration, wash the bladder enough for several times until all fulgurated white debris were completely cleaned out, because living tumor cells might still exist in the debris. As shown in the Table 1, although some patients recurred at other location, many of the treated patients showed tumor free for more than 6 months in the treated area. Patient \#3 suffered severe Alzheimer disease, and though tumor recurred several times, office-based treatment was beneficial for both patient's family and surgeon as much.

\section{Conclusion and Tips}

Office-based biopsy and fulguration for tiny bladder tumor is thought to be effective and less invasive procedure as a treatment of bladder cancer patients. Tips of the successful transurethral biopsy and fulguration for tiny bladder tumor are as follows: 1) sole lesion; 2) less than $5 \mathrm{~mm}$ in size; 3) wash bladder enough after the procedure with distilled water.

\section{REFERENCES}

[1] J. W. Akornor, J. W. Segura and A. Nehra, "General and 
Cystoscopic Procedures," Urologic Clinics of North America, Vol. 32, No. 3, 2005, pp. 319-326.

http://dx.doi.org/10.1016/j.ucl.2005.04.002

[2] B. B. O’Neil and W. T. Lowrance, “Office-Based Bladder Tumor Fulguration and Surveillance, Indications and Techniques," Urologic Clinics of North America, Vol. 40, No. 2, 2013, pp. 175-182. http://dx.doi.org/10.1016/j.ucl.2013.01.007

[3] A. W. Wedderburn, P. Ratan and B. R. Birch, “A Prospective Trial of Flexible Cystodiathermy for Recurrent Transitional Cell Carcinoma of the Bladder," Journal of Urology, Vol. 161, No. 3, 1999, pp. 765-776. http://dx.doi.org/10.1016/S0022-5347(01)61778-6

[4] S. M. Donat, A. North, G. Dalbagni and H. W. Herr, "Efficacy of Office Fulguration for Recurrent Low Grade
Papillary Bladder Tumors Less than $0.5 \mathrm{~cm}$," Journal of Urology, Vol. 171, No. 2, 2004, pp. 636-639. http://dx.doi.org/10.1097/01.ju.0000103100.22951.5e

[5] H. W. Herr, S. M. Donat and V. E. Reuter, "Management of Low Grade Papillary Bladder Tumors," Journal of Urology, Vol. 178, No. 4, 2007, pp. 1201-1205. http://dx.doi.org/10.1016/j.juro.2007.05.148

[6] W. E. Wysokinski and R. D. McBane II, "Periprocedural Bridging Management of Anticoagulation," Circulation, Vol. 126, 2012, pp. 486-490.

http://dx.doi.org/10.1161/CIRCULATIONAHA.112.0928 $\underline{33}$ 